\title{
INTERESTING CASES.
}

\section{Case I.}

A MARRIED woman, aged 37, was sent to hospital for an opinion. Her doctor said that for two years she had complained of slight cough, with increasing shortness of breath, but little or no expectoration. Latterly he had noticed that she had a certain amount of stridor. He had obtained with difficulty one specimen of sputum which was examined for the presence of T.B., with negative result. She was admitted for observation and her condition found to be as follows: The temperature was normal, the pulse a little more rapid than normal. The sputum was scanty ; no T.B. were found on examination. The Wassermann reaction was negative. On examination of the chest harsh breath sounds with prolonged expiration were heard all over both sides, accompanied by medium dry rhonchi. Bronchial breathing, somewhat lowpitched, was audible over the left upper zone of the chest, where there was marked dullness on percussion. The vocal resonance in this area was definitely increased. The heart was displaced towards the left. Slight stridor was noticeable.

$\mathrm{X}$-ray examination of the chest showed a diffuse opacity in the left upper zone, corresponding more or less to the left upper lobe, with a hard shadow at the left hilum suggestive of an old healed calcified tuberculous gland. There was no mottling of the lung area such as would have suggested tuberculous infiltration of the lung parenchyma, the opacity of the left upper lobe being, in the opinion of the radiologist, rather indicative of a condition of collapse secondary to bronchial obstruction, with possibly some fibrotic changes. There was a general increase of the linear striation from the roots of the lungs. The displacement of the heart towards the lett, already noted on clinical examination, was confirmed by the $\mathrm{X}$-ray. No diagnosis was made at this stage. The radiologist said at the time that he could not exclude the possibility of a neoplasn.

It was thought that thoracoscopic examination of the interior of the chest might yield information of value. A preliminary pneumothorax was accordingly induced on the left side, 400 c.c. of air being introduced on the first day, 400 c.c. on the second day, and 700 c.c. on the third day. On the fourth day, after introduction of a further 600 c.c. of air, the thoracoscope was employed and many dense adhesions were seen all over the upper part of the left pleural cavity. A few fine adhesions, like a spider's web, were visible at the extreme apex of the thoracic cavity, but the apex of the lung itself was seen to be free and moving well. A considerable surface of the visceral pleura was viewed, and in one place a small nodule was observed; there were also some suspicious looking areas which had an appearance like that of granulation tissue; these, it was thought, might possibly be scattered nodules of growth. The area in which they were visible was, however, very limited, and in view of the length of the patient's history (her symptoms were of two years' duration) it was felt that the diagnosis of pleurogenous new growth was somewhat doubtful. It was supposed, alternatively, that the mass seen in the hilum region in the skiagram was an old calcareous tuberculous gland which was causing pressure in the region of the bifurcation of the trachea, and giving rise to some stenosis of the bronchus with secondary atelectasis of the lung. It still, however, seemed a little difficult to account for the existence of such extensive fibrosis and of the numerous adhesions. 
This patient left the hospital and returned home shortly afterwards. About two months later she came again to report herself, saying that sh ewas feeling very much better and could breathe more comfortably and that movements of her left arm and shoulder were more free than before. On examination of the chest the air entry in the left upper zone was better than before, and there were now no adventitious sounds to be heard. It was supposed that the stretching of adhesions by the rather strenuous collapse of the lung must have had some effect in relieving her chest symptoms. At any rate her general condition was undoubtedly much improved, and her symptoms considerably less, though the exact pathology and diagnosis of her condition were still very much a matter of difficulty and conjecture. Nothing further was heard of this patient until some years later, when the following facts were given by ber doctor. Nearly four years after she was first seen at the hospital, she had an attack of acute pneumonia (sic) in the early part of the year, and from this time onwards she went steadily and rapidly downhill. The sputum, which was abundant, was found to contain enormous numbers of T.B. Signs of excavation developed and she died in the autumn from a very acute pulmonary tuberculosis. Apparently the adhesions and fibrous changes in the lung discovered on investigation four years previously must have been of tuberculous origin, the disease having remained quiescent for a considerable period and having finally been stirred into sudden activity with all the clinical manifestations of an acute tuberculous pneumonia. In view of the total length of the history of this case (six years) there seems no reason to suppose the co-existence of any pulmonary new growth which was at first suspected very strongly.

\section{Case II.}

A YOUNG man, aged 24, was admitted to hospital for observation and treatment, with a provisional diagnosis of gastric ulcer. He had been attended by his own doctor for about six months on account of pain in the upper part of the abdomen with frequent vomiting, usually immediately after meals. There had been no hæmatemesis or melæna ; the bowels were always constipated. An X-ray examination (barium meal) had been made and a report sent by the radiologist to the effect that the appearances were suggestive of the presence of an ulcer; the stomach appeared to empty with unusual rapidity.

This patient remained in hospital for five weeks and was treated on the usual lines -careful dieting and the administration of bismuth and alkalies-and at the end of this period he was discharged very much better, and after a short interval resumed his work. $\mathrm{He}$ continued to attend as an out-patient and to take a mixture of bismuth and pepsin after his meals. He continued in very fair health and without complaining of any marked symptoms for about a month, when one day he got chilled and wet while at work and returned home somewhat exhausted at the end of the day. On arrival at home he was seized with very severe abdominal pain which doubled him up (sic) and he vomited several times. After a very distressing night he came to the Out-patient Department on the following morning in considerable pain and looking desperately ill. He was blanched, as if he had lost a quantity of blood, and a good deal collapsed. He was admitted to an emergency bed at once.

On examination of the abdomen there was marked pain and tenderness in the 
epigastric region and also in the back on the left side low down (i.e., over an area extending from the angle of the scapula to the twelfth rib). There were no unusual physical signs at the base of the left lung behind. From the previous history and the onset of acute abdominal pain together with the extreme tenderness both in the back and in the epigastric region it was concluded that he had had a small perforation of a gastric ulcer with the formation of peri-gastric adhesions, and the possibility of leaking and the formation of a sub-phrenic abscess was discussed. The blood-picture was as follows :-

Total red cells, 2,500,000 ; Hæmoglobin, 50 per cent.; Colour index, r०o ; Total white cells, 18,000 .

Differential Leucocyte Count.-Polymorphonuclears, 69 per cent. ; Small lymphocytes, 20 per cent. ; Large mononuclears, 9 per cent. ; Eosinophiles, 2 per cent.

The Widal Reaction was negative to the Bacillus typhosus, to paratyphosus $a$, and paratyphosus $\beta$, in dilutions of $I$ in 400 to $I$ in 25 .

The pathologist reported that the blood-count was compatible with the occurrence of a severe hæmorrhage.

The temperature was $99^{\circ} 2^{\circ} \mathrm{F}$,, the pulse rate IIo. The pain and tenderness persisted and there was marked hyperæsthesia of the skin over the stomach area in front and also over an area behind below the left scapula and extending approximately from the level of the eighth thoracic vertebra to the level of the twelfth thoracic vertebra. The surgeon who was asked to see him in consultation was of the opinion that it would be wiser to wait for a little while before deciding to explore the abdominal cavity, and meanwhile endeavours were made to find a relation who was a suitable donor, it being thought that after blood transfusion the patient would have a better chance from the operation. As his condition appeared at first to improve somewhat, it was thought desirable still to wait, and he was carefully watched for some days. Vomiting occurred during one night, the vomited matter containing no blood, but a small quantity of pus. After a further interval the tenderness in front and behind increased. No swelling or localizing evidence of a collection of pus having developed, it was decided to open the abdomen, since the patient was getting obviously worse, and it was thought that he was bleeding into the abdominal cavity.

A median laparotomy was performed. This revealed a large inoperable mass of carcinomatous growth involving the whole of the fundus and cardiac end of the stomach. No obstruction having occurred there was no indication for a short-circuit operation and the abdomen was closed. The patient died a fortnight later.

Case III.

A BоY, aged 6, was admitted to hospital for immediate operation on the assumption that he was suffering from acute infective arthritis of the hip-joint, probably due to acute osteo-myelitis of the neck of the femur. For three days he had complained of pain and stiffness in the hip, with increasing difficulty on walking. He had not vomited.

On examination he was obviously ill, with a temperature of $\operatorname{ror}^{\circ} 2^{\circ} \mathrm{F}$., and a pulse rate of 122 . The right hip was rigidly flexed at an angle of about $30^{\circ}$, and any attempt to increase flexion or to extend the joint was tearfully resented. A more detailed examination revealed a fixed, indurated and tender swelling above Poupart's ligament. 
Rotary movements of the hip could be obtained painlessly, which disproved the diagnosis of acute arthritis. No obvious source of infection could be discovered.

Diagnosis.-Adenitis of the right external iliac glands. Acute arthritis of the hip joint was suggested by the presence of psoas spasm, but was subsequently refuted by the discovery that rotation could be oblained. The fact that no source of infection was found is explained by the assumption that the causative focus was small and had cleared up in the interval. Six days after admission an abscess was evacuated, and recovery was uneventful.

As in other parts of the body, infection may "jump" one group of glands, and affect those more central, which explains why the inguinal glands were not obviously enlarged.

\section{Case IV.}

A mAN, aged 39, was admitted to hospital and stated that he had suffered from leftsided abdominal discomfort for five days. He had found walking increasingly difficult, but by leaning forwards he could still walk slowly. The pain increased in intensity, so he took to his bed and sent for his doctor, who found a tender swelling in the left iliac fossa. The doctor sent him to hospital with a diagnosis of diverticulitis and pericolic infection. On admission his temperature was $102.2 \mathrm{~S} F$. and pulse-rate 108, and a rounded swelling the size of a Jaffa orange was palpable above the left Poupart's ligament. The swelling was tender and indefinite in outline. There was no previous history of any digestive disturbances, and his bowels were and had been, open regularly.

Diagnosis,-Adenitis of the left external iliac glands. The entire absence of any abdominal symptoms discounted the diagnosis of intra-peritoneal inflammation. The correct diagnosis was suggested by the presence on both ankles of numerous infected gnat bites and by the psoas spasm. Two days after admission the swelling had increased in size and was obviously adherent to the abdominal wall. Half a pint of pus was evacuated from which staphylococci were isolated. Recovery was uneventful.

\section{CASES FOR DIAGNOSIS.}

\section{Case I.}

A Woman, aged 30, no children, past history negative; menstruation regular every twenty-eight days, lasting four days, last regular period ten weeks ago, comes up complaining of severe abdominal pain and inability to void urine.

On examination the temperature is normal, pulse 90 ; there is per abdomen a very tender elastic pyriform swelling extending from the symphisis pubis to the umbilicus. Per vaginam the cervix is directed forward lying behind the pubic symphisis; in the posterior fornix can be felt an ill-defined, softly elastic swelling, somewhat tender and apparently filling the pelvis.

How would you proceed to investigate this case? What is the diagnosis and treatment? 